\title{
Loosely Coupled Dominating Set for Cluster-Based Wireless Sensor/Mesh Networks
}

\author{
Chie Dou* and Yu-Hui Chang \\ Department of Electrical Engineering, National Yunlin University of Science and Technology \\ No. 123, Section 3, University Road, 640 Touliu, Yunlin, Taiwan \\ (Received July 2, 2014; accepted April 2, 2015)
}

Key words: connected dominating set, wireless sensor/mesh networks, clustering approach, node utilization, edge coverage

The clustering approach is one of the most promising solutions for establishing virtual backbones in wireless sensor/mesh networks (WSN/WMNs). The most basic network clustering structure that has been investigated is based on dominating sets. Usually, nodes in a dominating set consume more energy than nodes outside the set. A simple mechanism to prolong the life cycle of each node in the network by balancing their energy consumption is to rotate the active dominating set through a set of disjoint dominating sets. In this paper, we propose a novel loosely coupled dominating set (LCDS) for cluster-based WSN/WMNs, which is adapted from the concept of a weakly connected dominating set (WCDS) by further relaxing its connectivity requirement. The main advantages of the proposed LCDS are as follows. First, it decreases the average utilization of the chosen cluster heads to prolong their life cycles. Second, it increases the edge coverage of the network to exploit the benefits of load balancing. Third, it provides the network with higher reliability by supporting more alternative routing paths. Performance comparisons between the LCDS and the WCDS are given in a rotation scenario with three disjoint dominating sets. Numerical results show that the proposed LCDS outperforms the WCDS in every node deployment scenario and every performance aspect.

\section{Introduction}

In a dominating set (DS)-based network clustering structure, a particular vertex called cluster head $(\mathrm{CH})$ within each cluster may be designated to oversee channel allocation and message routing. A node set is a dominating set if every node in the network is either in the set or a neighbor of a node in the set. A connected dominating set (CDS), say D,

${ }^{*}$ Corresponding author: e-mail: douc@yuntech.edu.tw 
of a graph $G=(V, E)$ is a set of vertices with two properties: (1) $D$ is a dominating set in $G$ and (2) $D$ induces a connected subgraph of $G$. Messages can be routed from the source node to a neighbor in the dominating set, along the CDS to the dominating set member closest to the destination node, and then finally to the destination. ${ }^{(1)}$ Message routing via the CDS results in a significant reduction in message overhead associated with routing updates. ${ }^{(2)}$ Although the CDS provides an obvious virtual backbone for message routing, the connectivity requirement causes the size of CDS to be large. Relaxing this requirement can reduce the size effectively. A minimum CDS (MCDS) is a CDS that has the minimum number of nodes. Many researchers have focused on MCDS-based virtual backbone construction in the past years. ${ }^{(3-5)}$ The weakly connected dominating set (WCDS) is another approach that can reduce the size effectively. A WCDS is a node set that is dominating, and all the edges with at least one end point in the set form a connected subgraph (weakly induced subgraph). Using an underlying cluster-based virtual backbone induced by the WCDS can be a very promising approach to enhance network efficiency. ${ }^{(6-8)}$ The problem of finding a CDS or an MCDS or a WCDS is NPhard (nondeterministic polynomial-time hard).

In this paper, we propose a novel loosely coupled dominating set (LCDS), which is adapted from the concept of WCDS by further relaxing its connectivity requirement. Figure 1(a) shows a typical WCDS formation in a small cellular scenario. Clearly, there is a white node (named bridge node) between two neighboring black nodes (CHs). That is, the hop count between two neighboring $\mathrm{CHs}$ is only two with the node pattern B-W-B (black-white-black). Figure 1(b) shows a typical LCDS formation in the same cellular scenario. Evidently, the LCDS is also a dominating set in that every node in the network is indeed either in the set or a neighbor of a node in the set. However, the hop count between two neighboring $\mathrm{CHs}$ now becomes three with the node pattern B-WW-B (black-white-white-black). In this study, we use a simple method of differentiating between the WCDS and the LCDS formation algorithms. For the WCDS algorithm in this simple method, the next $\mathrm{CH}$ is selected from the two-hop neighboring nodes of a previously chosen $\mathrm{CH}$; for the LCDS algorithm, the selection criterion is relaxed by allowing the next $\mathrm{CH}$ to be selected from the two-hop or three-hop neighboring nodes

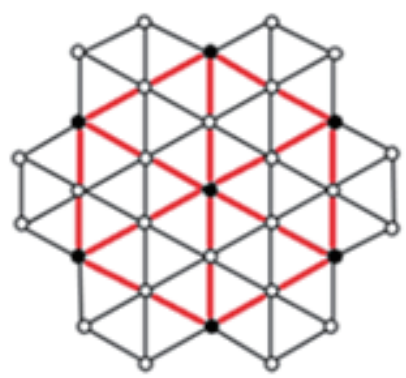

(a)

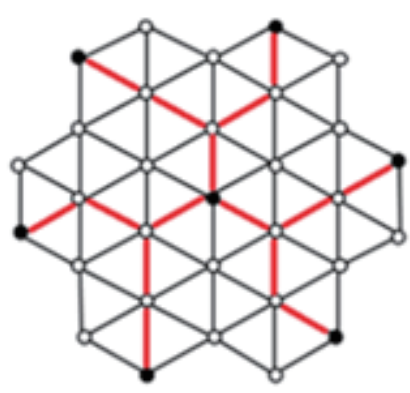

(b)

Fig. 1. (Color online) (a) An example of WCDS. (b) An example of LCDS. 
of a previously chosen $\mathrm{CH}$. The main advantages of the proposed LCDS algorithm are as follows. First, it can further reduce the size of the dominating set compared with that generated by the WCDS. Second, it can decrease the average utilization of the chosen $\mathrm{CHs}$ to prolong their life cycle. Third, it can increase the edge coverage of the network to exploit the benefits of load balancing. Fourth, it provides the network with higher reliability by supporting more alternative routing paths.

Nodes in a dominating set usually consume more energy in order to handle extra load of various bypass traffics than nodes outside the set. A simple mechanism to prolong the life cycle of each node in the network by balancing their energy consumption is to rotate the active dominating set through a set of disjoint dominating sets. ${ }^{(9,10)}$ In this paper, a disjoint WCDS/LCDS formation algorithm is introduced. Performance comparisons between the LCDS and the WCDS are given in a rotation scenario with three disjoint dominating sets. Numerical results show that the proposed LCDS outperforms the WCDS in every node deployment scenario and every performance aspect.

\section{Disjoint WCDS/LCDS Formation Algorithm}

\section{$2.1 \quad W C D S / L C D S$ formation algorithm}

The WCDS algorithm used in this paper was adapted from a centralized WCDS formation algorithm proposed in ref. 6. The detailed steps of our WCDS/LCDS formation algorithm are described as follows.

Step 1: Initially, all nodes are colored white.

Step 2: Find the node with the maximum number of one-hop adjacent nodes; set this node to be the starting point and be the first chosen $\mathrm{CH}$ (marked in black); then mark all its one-hop neighboring nodes in gray.

Step 3: Find the node with the maximum number of one-hop adjacent nodes from the two-hop neighboring nodes of the first chosen $\mathrm{CH}$ for WCDS formation, or from the two-hop or three-hop neighboring nodes of the first chosen $\mathrm{CH}$ for LCDS formation, and set it to be the next chosen $\mathrm{CH}$; then, mark all its one-hop adjacent nodes in gray.

Step 4: Find the node with the maximum number of one-hop adjacent nodes from all the already chosen CHs' two-hop neighboring nodes for WCDS formation, or from all the already chosen CHs' two-hop or three-hop neighboring nodes for LCDS formation, and set it to be the next chosen $\mathrm{CH}$; then, mark all its onehop neighboring nodes in gray. Keep on doing so until all the nodes are marked either in black or gray.

Dominating set formation examples of the WCDS and LCDS by using the above algorithm are shown in Figs. 2(a) and 2(b), respectively. In Fig. 2, 110 nodes labeled with their node IDs are randomly deployed in a 9 by 6 rectangular area. All the nodes in the graph have the same transmission radius 1 . The node density of the graph, defined as the average number of one-hop adjacent nodes of all nodes, is 4.7. A total of $24 \mathrm{CHs}$ were selected out of the 110 nodes in Fig. 2(a). In Fig. 2(b), the number of CHs selected from the 110 nodes is now reduced to 23 . 


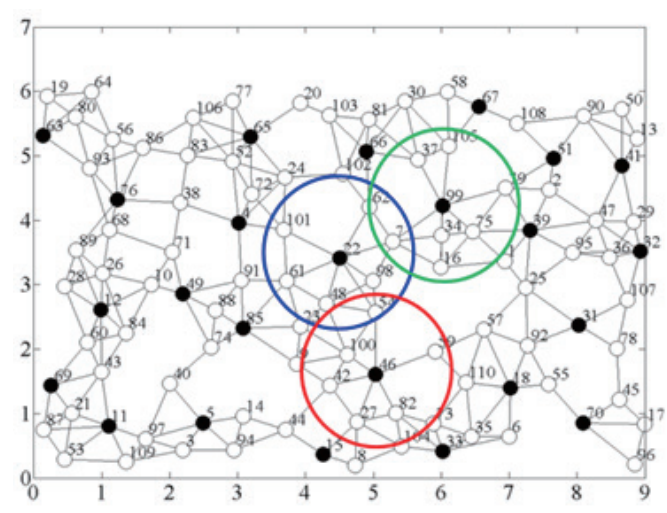

(a)

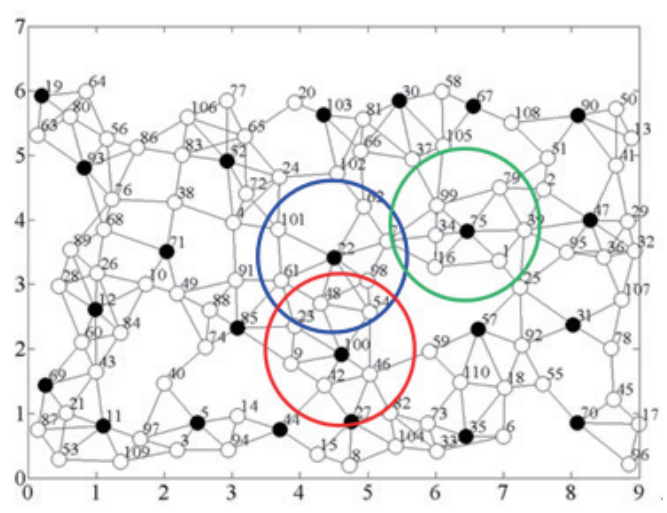

(b)

Fig. 2. (Color online) (a) A WCDS formation example. (b) An LCDS formation example.

\subsection{Disjoint WCDS/LCDS formation algorithm}

Let $D N$ denote the total number of disjoint sets that the algorithm attempted to find out, and let Iter_Num be the iteration number of the algorithm currently being executed. For example, $D N=3$ and Iter_Num $=2$ denote that the algorithm is now attempting to find the second disjoint WCDS or LCDS, and the remaining number of disjoint sets that the algorithm needs to continue to find, denoted by Rem_Dis_Num, which is equal to DN minus Iter_Num, is 1 . Any white node $\mathrm{u}$ can be chosen as a new $\mathrm{CH}$ by the algorithm only if the following "New_CH_Candidate" criterion is satisfied. First, so far, node u has not been selected as a $\overline{\mathrm{C}} \mathrm{H}$ by the algorithm. Second, the inequality listed below is satisfied.

$$
\text { Num_lH(u) - Num_CH_lH(u) } \geq \text { Rem_Dis_Num, }
$$

where Num_1H(u) represents the number of one-hop neighboring nodes of node $u$, and Num_CH_ $\overline{1 H}(u)$ represents the number of one-hop neighboring nodes of node $u$ that were already chosen as $\mathrm{CHs}$ in the previous algorithm iterations.

The detailed steps of the disjoint WCDS/LCDS formation algorithm are described as follows.

Initial setting: Iter_Num $=1$

Step 1: Compute Rem_Dis_Num $=$ DN - Iter_Num.

Step 2: Initially, all nodes are colored white.

Step 3: Find the node with the maximum number of one-hop adjacent nodes and satisfies the "New_CH_Candidate" criterion; set this node to be the starting point and be the first chosen $\mathrm{CH}$ (marked in black) in the current iteration; then mark all its one-hop neighboring white nodes in gray.

Steps 4 and 5: These two steps are the same as that presented in steps 3 and 4 of the previous WCDS/LCDS formation algorithm, except that the newly selected $\mathrm{CH}$ must satisfy the "New_CH_Candidate" criterion. 
Step 6: Supplementary procedure: If some white nodes still exist after step 5 has been completed, a supplementary $\mathrm{CH}$ has to be chosen for each of these white nodes from its one-hop neighboring white nodes, which satisfy the "New_CH Candidate" criterion. If the supplementary procedure cannot be completed, the algorithm is stopped and the current iteration is abandoned, such that only Iter Num -1 sets of WCDS/LCDS can be found.

Step 7: The current iteration is completed when all the nodes are marked either in black or gray. If Iter_Num++ is greater than $D N$, then stop the algorithm (i.e., all disjoint WCDSs/LCDSs are found successfully); otherwise, go to step 1 for the next iteration.

\section{Performance Indicators}

The following performance indicators, namely, dominating set size, average $\mathrm{CH}$ utilization, average bridge utilization, total edge coverage, and average $\mathrm{CH}$-to- $\mathrm{CH}$ hop count, are used for the comparison between the performances of the WCDS and the LCDS.

\subsection{Virtual cluster (VC)-based shortest path routing}

A $\mathrm{VC}$ is a set consisting of a $\mathrm{CH}$ and all its associated one-hop and two-hop neighboring nodes, in which the $\mathrm{CH}$ serves as the center and the coordinator of the $\mathrm{VC}$. The $\mathrm{CH}$ has to coordinate all data-exchange requests issued by its one-hop neighbors. If the destination node of a data-exchange request is not within the coverage of a VC (i.e., the destination node is beyond the two-hop distance from the $\mathrm{CH}$ of the $\mathrm{VC}$ ), then the $\mathrm{CH}$ will recognize it as an Inter-VC routing. All Inter-VC shortest routing paths can be obtained by constructing a spanning tree from the $\mathrm{CH}$ of the source $\mathrm{VC}$ to the $\mathrm{CH}$ of every other VC. Figure 3(a) shows a well-constructed spanning tree example for the

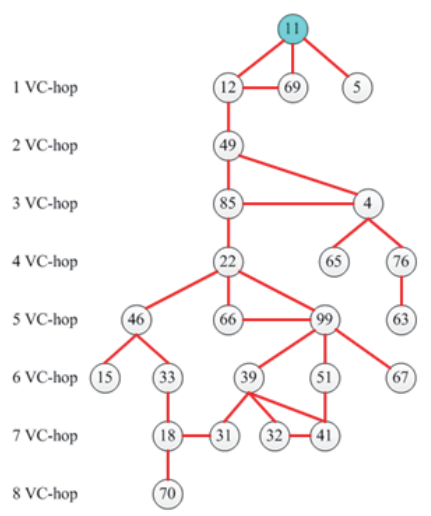

(a)

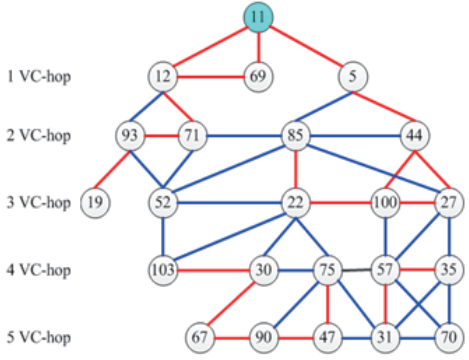

(b)

Fig. 3. (Color online) (a) A spanning tree example of the WCDS. (b) A spanning tree example of the $\mathrm{LCDS}$. 
WCDS shown in Fig. 2(a), using VC_11 as the source VC. In Fig. 3(a), we use VC-hop count to measure the distance between the source $\mathrm{VC}$ and the destination $\mathrm{VC}$. To build the shortest path between two CHs in the spanning tree shown in Fig. 3(a), one must follow the WCDS node pattern B-W-B in every VC-hop. In the spanning tree example of the LCDS shown in Fig. 3(b), one may follow the LCDS node patterns B-W-B or B-W-W-B in every VC-hop to build the shortest path. In Fig. 3(b), each VC-hop in the spanning tree could be colored in red or blue. A red line means that the node pattern between two adjacent $\mathrm{CHs}$ is $\mathrm{B}-\mathrm{W}-\mathrm{B}$, which is a two-node hop. A blue line implies that the node pattern between two adjacent $\mathrm{CHs}$ is $\mathrm{B}-\mathrm{W}-\mathrm{W}-\mathrm{B}$, which is a three-node hop.

In Fig. 3(b), it is worth noting that there are three routing paths from node 11 to node 52 with three VC-hops. The shortest one is $11 \rightarrow 12 \rightarrow 71 \rightarrow 52$ with two red lines $(11 \rightarrow 12$, $12 \rightarrow 71)$ and one blue line $(71 \rightarrow 52)$. If this path fails, there still remain two alternative routes in the LCDS to choose from. Thus, by supporting more alternative routing paths, the LCDS provides the network with higher reliability than the WCDS. Also, we observed that the depth of the VC-hop count of the spanning tree shown in Fig. 3(b) is smaller than that shown in Fig. 3(a). It means that the LCDS can provide shorter routing path(s) between two CHs than the WCDS. This can surely decrease the utilization of the CHs.

\subsection{Formulas for calculating performance indicators}

We use the shortest path between two CHs, nodes 11 and 52, depicted above to illustrate how to compute these performance indicators for the WCDS and the LCDS. The shortest path $11 \rightarrow 12 \rightarrow 71 \rightarrow 52$ gives the following information. First, four CHs are traversed by the path: nodes $11,12,71$, and 52 ; all have been used only once. Second, seven edges are traversed by the path: $11 \rightarrow 43,43 \rightarrow 12,12 \rightarrow 10,10 \rightarrow 71,71 \rightarrow 38$, $38 \rightarrow 83$, and $83 \rightarrow 52$; all have been used only once. Third, the VC-hop count traversed by the path is 3 , and the node-hop count is $7(2+2+3)$. The total frequency of use of each node, either black or white, can be computed by counting the number of shortest paths that traverse this node. The total frequency of use of each edge can be computed similarly. The total number of shortest paths in a graph is equal to the total number of black node pairs (any node pair between two CHs). The utilization of a node $u$, denoted by $U_{u}$, is computed as follows:

$$
U_{u}=\frac{\text { Frequency of use of node } u}{\text { Total number of shortest paths in the graph }} .
$$

The average utilization of a $\mathrm{CH}$, denoted as $\bar{U}_{\mathrm{CH}}$, is given by

$$
\bar{U}_{\mathrm{CH}}=\frac{\sum_{\text {for all } \mathrm{CHs}} U_{\mathrm{CH}}}{\text { Total number of } \mathrm{CHs}} \text {. }
$$

The average utilization of a bridge node, denoted as $\bar{U}_{\text {Bridge }}$, is given by

$$
\bar{U}_{\text {Bridge }}=\frac{\sum_{\text {for all bridges }} U_{\text {Bridge }}}{\text { Total number of bridges }} .
$$


The edge coverage of a graph, denoted by $C_{\text {edge }}$, is defined as

$$
C_{\text {edge }}=\frac{\text { Total number of edges being used }}{\text { Total number of edges }} .
$$

Since performance comparisons between the LCDS and the WCDS are given in a rotation scenario with three disjoint dominating sets, the total frequency of use of a node or of an edge should be the summation of the frequency of use of that node or of that edge in every individual dominating set operating environment.

\section{Performance Comparisons}

Performance comparisons between the LCDS and the WCDS are discussed in a rotation scenario with three disjoint dominating sets. The proposed disjoint WCDS/ LCDS formation algorithm is used to find the three disjoint WCDSs and LCDSs. The first disjoint WCDS and LCDS, as that shown in Figs. 2(a) and 2(b), are redrawn in Figs. 4(a) and 4(b), respectively. There are two differences between the original figures and the redrawn figures. First, the label of each node is changed from its node ID to the frequency of use of that node. Second, each edge is colored differently in the graphs shown in Figs. 4(a) and 4(b) according to the frequency of use of that edge. As shown in Table 1, six levels of degree with different colors and widths are used to mark each edge. The darker and wider the edge, the higher the frequency of use of that edge. If the frequency of use of the edge is 0 , the edge is colored green. Figures 4(c) and 4(d) show the first two disjoint WCDSs and LCDSs, respectively. The CHs in the first WCDS/ LCDS are colored black, and the CHs in the second WCDS/LCDS are colored blue. Figures 4(e) and 4(f) show the three disjoint WCDSs and LCDSs, respectively. The CHs in the third WCDS/LCDS are colored green.

The performance indexes in the rotation scenario with three disjoint WCDSs and LCDSs are shown in Table 2. Numerical results show that the LCDS has a smaller DS size, a lower average $\mathrm{CH}$ and average bridge utilizations, and a higher ratio of cumulative edge coverage than the WCDS. The total frequency of use of each node in the three disjoint WCDS/LCDS rotation scenario is shown in Fig. 5. Evidently, the WCDS has more nodes with heavy usage than the LCDS. The cumulative edge coverage of the three disjoint WCDSs is increased from $31 \%$ to $50 \%$ and then to $70 \%$. However, that of the three disjoint LCDSs performs better; it can be improved from $55 \%$ to $83 \%$ and then to $92 \%$. The higher ratio of the edge coverage means that the traffic load is more evenly distributed among all the edges. This also explains why there are more green edges and bolder edges colored in red in the graphs shown in Figs. 4(c) and 4(e) than in those shown in Figs. 4(d) and 4(f).

The average $\mathrm{CH}$-to- $\mathrm{CH}$ node-hop counts of the three disjoint WCDSs have individual values of 7.4, 8.8, and 8.1, and that of the three disjoint LCDSs have individual values of 6.9, 6.9, and 6.7. This result is consistent with the previous observation that the depth of the VC-hop count of the spanning tree shown in Fig. 3(b) is smaller than that shown in Fig. 3(a). Thus, the LCDS has a smaller average CH-to-CH node-hop count than the WCDS. 


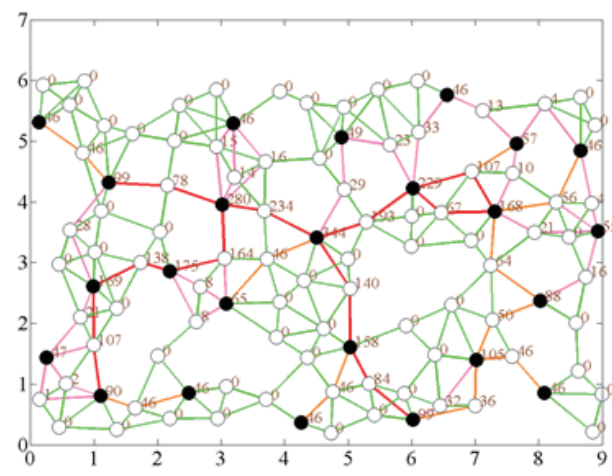

(a)

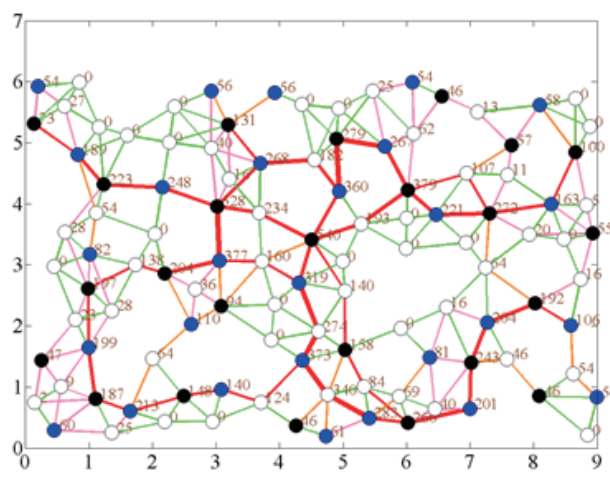

(c)

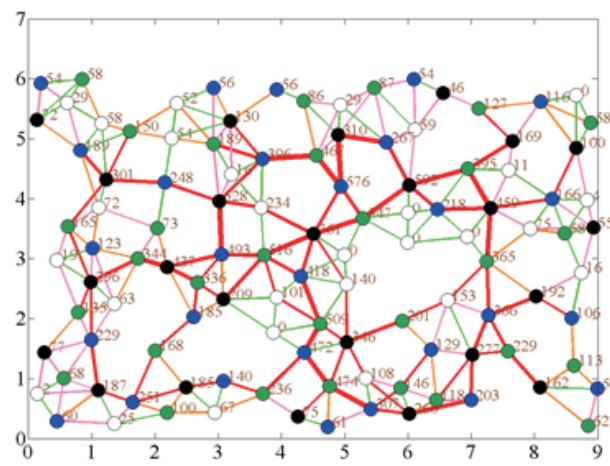

(e)

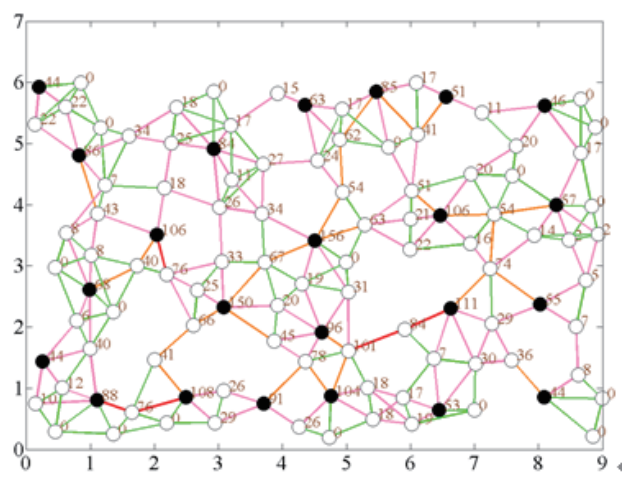

(b)

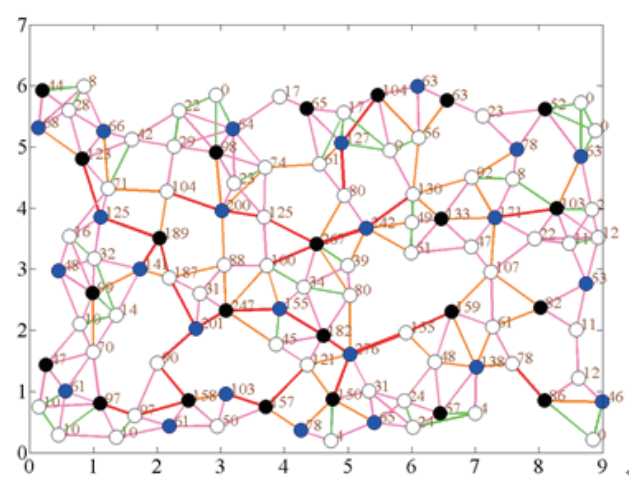

(d)

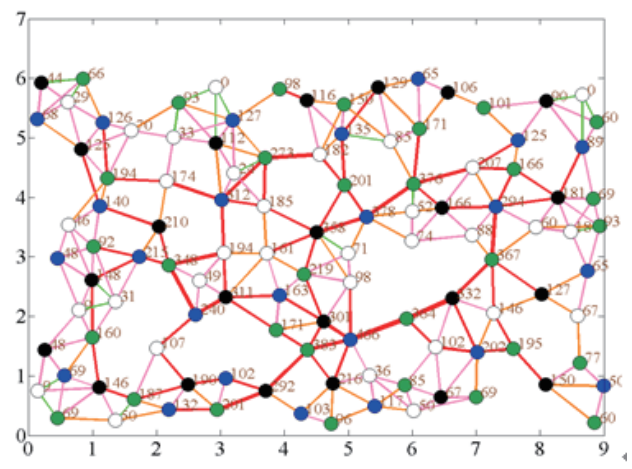

(f)

Fig. 4. (Color online) (a) 1st disjoint WCDS. (b) 1st disjoint LCDS. (c) Two disjoint WCDSs. (d) Two disjoint LCDSs. (e) Three disjoint WCDSs. (f) Three disjoint LCDSs. 
Table 1

Six levels of degree with different colors and widths.

\begin{tabular}{lcccccc}
\hline \multirow{2}{*}{ Color } & \multirow{2}{*}{ Green } & \multirow{2}{*}{ Pink } & \multirow{2}{*}{ Orange } & \multicolumn{3}{c}{ Red } \\
\cline { 5 - 7 } & & & & Thin & Intermediate & Bold \\
\hline Freq. of use & 0 & $0-34$ & $35-69$ & $70-149$ & $150-249$ & $>250$ \\
\hline
\end{tabular}

Table 2

Performance indexes in the rotation scenario with three disjoint sets.

\begin{tabular}{|c|c|c|c|c|}
\hline & DS size & $\begin{array}{c}\text { Avg. } \mathrm{CH} \\
\text { utilization }\end{array}$ & $\begin{array}{l}\text { Avg. bridge } \\
\text { utilization }\end{array}$ & $\begin{array}{c}\text { Cumulative } \\
\text { edge coverage }\end{array}$ \\
\hline WCDS & $24 \quad 28 \quad 30$ & $20 \% \quad 19 \% \quad 17 \%$ & $10 \% \quad 12 \% \quad 10 \%$ & $31 \% \quad 50 \% \quad 70 \%$ \\
\hline LCDS & $24 \quad 31$ & $16 \% \quad 15 \% \quad 12 \%$ & $6 \%$ & $55 \% \quad 83 \% \quad 92 \%$ \\
\hline
\end{tabular}

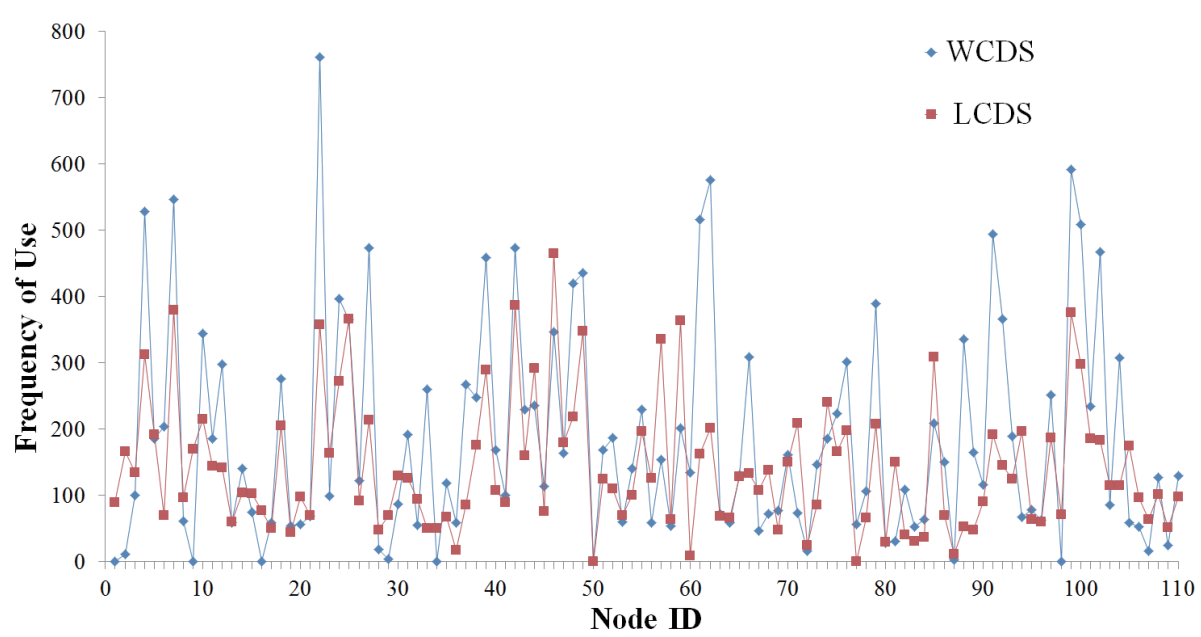

Fig. 5. (Color online) Total frequency of use of each node in the rotation scenario with three disjoint dominating sets.

Thorough performance comparisons between the WCDS and the LCDS in a three disjoint dominating sets rotation scenario are given in Tables 3 and 4, under different graph sizes from 100 to 200 nodes with the same graph density equal to 6 . The same conclusions as those obtained from Fig. 4 and Table 2 can be derived from Tables 3 and 4. The cumulative edge coverage of the three disjoint LCDSs is increased from about $55 \%$ to $80 \%$ and then to $90 \%$ under all graph sizes. The average $\mathrm{CH}$-to- $\mathrm{CH}$ node-hop counts of all three disjoint WCDSs are about 7-8 for graph sizes from 100 to 150 and about 8-10 for graph sizes from 160 to 200 . The LCDS has a smaller average $\mathrm{CH}$-to-CH node-hop count; the values are about 6-7 for graph sizes from 100 to 150 and about 7-8 for graph sizes from 160 to 200 . 
Table 3

Performance indexes of the WCDS under different graph sizes.

\begin{tabular}{lllllllllllll}
\hline $\begin{array}{l}\text { Graph } \\
\text { size }\end{array}$ & \multicolumn{3}{c}{ DS size } & \multicolumn{4}{c}{$\begin{array}{c}\text { Avg. CH } \\
\text { utilization }\end{array}$} & \multicolumn{2}{c}{$\begin{array}{c}\text { Avg. bridge } \\
\text { utilization }\end{array}$} & \multicolumn{3}{c}{$\begin{array}{c}\text { Cumulative } \\
\text { edge coverage }\end{array}$} \\
\hline 100 & 21 & 24 & 25 & $20 \%$ & $18 \%$ & $18 \%$ & $7 \%$ & $6 \%$ & $7 \%$ & $32 \%$ & $58 \%$ & $74 \%$ \\
110 & 23 & 24 & 28 & $19 \%$ & $21 \%$ & $16 \%$ & $7 \%$ & $9 \%$ & $7 \%$ & $28 \%$ & $49 \%$ & $66 \%$ \\
120 & 24 & 24 & 27 & $19 \%$ & $19 \%$ & $17 \%$ & $8 \%$ & $8 \%$ & $7 \%$ & $25 \%$ & $44 \%$ & $63 \%$ \\
130 & 28 & 28 & 30 & $16 \%$ & $18 \%$ & $18 \%$ & $6 \%$ & $7 \%$ & $8 \%$ & $31 \%$ & $52 \%$ & $69 \%$ \\
140 & 28 & 34 & 35 & $18 \%$ & $14 \%$ & $15 \%$ & $8 \%$ & $6 \%$ & $7 \%$ & $25 \%$ & $53 \%$ & $71 \%$ \\
150 & 33 & 31 & 32 & $14 \%$ & $15 \%$ & $16 \%$ & $6 \%$ & $7 \%$ & $7 \%$ & $32 \%$ & $50 \%$ & $65 \%$ \\
160 & 31 & 33 & 39 & $19 \%$ & $16 \%$ & $13 \%$ & $9 \%$ & $7 \%$ & $5 \%$ & $24 \%$ & $44 \%$ & $66 \%$ \\
170 & 33 & 36 & 39 & $15 \%$ & $14 \%$ & $12 \%$ & $6 \%$ & $6 \%$ & $5 \%$ & $26 \%$ & $46 \%$ & $65 \%$ \\
180 & 36 & 38 & 43 & $15 \%$ & $14 \%$ & $14 \%$ & $6 \%$ & $6 \%$ & $6 \%$ & $26 \%$ & $48 \%$ & $65 \%$ \\
190 & 40 & 44 & 42 & $13 \%$ & $12 \%$ & $16 \%$ & $6 \%$ & $5 \%$ & $7 \%$ & $28 \%$ & $52 \%$ & $68 \%$ \\
200 & 43 & 41 & 46 & $12 \%$ & $13 \%$ & $13 \%$ & $5 \%$ & $5 \%$ & $5 \%$ & $31 \%$ & $52 \%$ & $67 \%$ \\
\hline
\end{tabular}

Table 4

Performance indexes of the LCDS under different graph sizes.

\begin{tabular}{lllllllllllll}
\hline $\begin{array}{l}\text { Graph } \\
\text { size }\end{array}$ & \multicolumn{3}{c}{ DS size } & \multicolumn{4}{c}{$\begin{array}{c}\text { Avg. CH } \\
\text { utilization }\end{array}$} & \multicolumn{2}{c}{$\begin{array}{c}\text { Avg. bridge } \\
\text { utilization }\end{array}$} & \multicolumn{2}{c}{$\begin{array}{c}\text { Cumulative } \\
\text { edge coverage }\end{array}$} \\
\hline 100 & 19 & 23 & 24 & $17 \%$ & $15 \%$ & $14 \%$ & $5 \%$ & $5 \%$ & $5 \%$ & $49 \%$ & $78 \%$ & $91 \%$ \\
110 & 24 & 24 & 25 & $14 \%$ & $14 \%$ & $15 \%$ & $5 \%$ & $5 \%$ & $5 \%$ & $53 \%$ & $79 \%$ & $90 \%$ \\
120 & 23 & 25 & 27 & $15 \%$ & $14 \%$ & $14 \%$ & $4 \%$ & $5 \%$ & $5 \%$ & $49 \%$ & $77 \%$ & $88 \%$ \\
130 & 24 & 26 & 31 & $14 \%$ & $14 \%$ & $13 \%$ & $5 \%$ & $4 \%$ & $4 \%$ & $51 \%$ & $76 \%$ & $90 \%$ \\
140 & 29 & 33 & 35 & $13 \%$ & $12 \%$ & $11 \%$ & $5 \%$ & $4 \%$ & $4 \%$ & $57 \%$ & $83 \%$ & $93 \%$ \\
150 & 32 & 31 & 30 & $12 \%$ & $12 \%$ & $12 \%$ & $4 \%$ & $4 \%$ & $4 \%$ & $54 \%$ & $75 \%$ & $86 \%$ \\
160 & 32 & 31 & 36 & $12 \%$ & $13 \%$ & $11 \%$ & $4 \%$ & $4 \%$ & $4 \%$ & $56 \%$ & $80 \%$ & $91 \%$ \\
170 & 32 & 34 & 37 & $12 \%$ & $11 \%$ & $11 \%$ & $4 \%$ & $4 \%$ & $4 \%$ & $52 \%$ & $75 \%$ & $86 \%$ \\
180 & 35 & 37 & 40 & $11 \%$ & $10 \%$ & $10 \%$ & $4 \%$ & $4 \%$ & $4 \%$ & $54 \%$ & $77 \%$ & $91 \%$ \\
190 & 37 & 41 & 40 & $11 \%$ & $10 \%$ & $11 \%$ & $4 \%$ & $4 \%$ & $3 \%$ & $55 \%$ & $80 \%$ & $91 \%$ \\
200 & 38 & 36 & 47 & $11 \%$ & $11 \%$ & $9 \%$ & $3 \%$ & $3 \%$ & $3 \%$ & $53 \%$ & $77 \%$ & $91 \%$ \\
\hline
\end{tabular}

\section{Conclusions}

In this paper, we propose a novel LCDS for cluster-based WSN/WMNs to improve network performances including longer life cycle, better load balancing, and higher routing efficiency. The shortest path between two CHs of the LCDS can built on either B-W-B or B-W-W-B node patterns. Performance comparisons between the LCDS and the WCDS are given in a three disjoint dominating sets rotation scenario under different graph sizes. A disjoint WCDS/LCDS formation algorithm is presented in this paper. Numerical results show that the proposed LCDS outperforms the WCDS in every node deployment scenario and every performance aspect. The benefits of the LCDS can be summarized as follows. First, it may have less DS size than the WCDS. Second, the 
average CH/Bridge utilization of the LCDS is smaller than that of the WCDS under different graph sizes. Third, it increases the cumulative edge coverage to $90 \%$ in the three disjoint dominating sets rotation scenario as compared with that of $70 \%$ of the WCDS. Fourth, the LCDS has a smaller average $\mathrm{CH}$-to-CH node-hop count than the WCDS, and it can also provide the network with high reliability by supporting more alternative routing paths.

\section{Acknowledgements}

This work was supported by the National Science Council, Taiwan, under Grant no. NSC 102-2221-E-224-006.

\section{References}

1 J. Blum, M. Ding, A. Thaeler and X. Cheng: Connected Dominating Set in Sensor Networks and MANETs, Handbook of Combinatorial Optimization, eds. D. Z. Du and P. Pardalos (Kluwer Academic Publishers, Dordrecht, 2004) p. 329.

2 Y L. Chang and C. C. Hsu: Mobile Networks Appl. 5 (2000) 27.

3 Y. Li, M. T. Thai, F. Wang, C. Yi, P. J. Wan and D. Z. Du: Wireless Commun. Mobile Comput. 5 (2005) 927.

4 S. Funke, A. Kesselman and U. Meyer: ACM Trans. Sens. Networks 2 (2006) 444.

5 H. Du, W. Wu, Q. Ye, D. Li, W. Lee and X. Xu: IEEE Trans. Parallel Distrib. Syst. 24 (2013) 652.

6 Y. P. Chen and A. L. Liestman: Proc. 3rd Mobile Ad Hoc Networking \& Computing Symp. (ACM, 2002) p. 165.

7 N. Chakchouk, B. Hamdaoui and M. Frikha: Wireless Commun. Mobile Comput. 12 (2012) 195.

8 B. Han and W. Jia: J. Parallel Distrib. Comput. 67 (2007) 727.

9 T. Acharya, S. Chattopadhyay and R. Roy: Proc. Int. Conf. Inf. Commun. Technol. (IEEE, 2007) p. 336.

10 R. Misra and C. Mandal: IEEE Trans. Mobile Comput. 8 (2009) 488. 